\title{
Diffusion of oxygen molecules in fluorine-doped amorphous $\mathrm{SiO}_{2}$
}

Koichi Kajihara, ${ }^{1,2 *}$ Taisuke Miura, ${ }^{3}$ Hayato Kamioka, ${ }^{4}$

Masahiro Hirano, ${ }^{1,6}$ Linards Skuja, ${ }^{1,5}$ and Hideo Hosono ${ }^{1,6}$

1 Transparent Electro-Active Materials Project,

ERATO-SORST, Japan Science and Technology Agency,

in Frontier Research Center, S2-13,

Tokyo Institute of Technology, 4259 Nagatsuta,

Midori-ku, Yokohama 226-8503, Japan

2 Department of Applied Chemistry,

Graduate School of Urban Environmental Sciences, Tokyo Metropolitan University,

1-1 Minami-Osawa, Hachioji 192-0397, Japan

3 Research $\&$ Development Division, OMRON Laserfront Inc.,

1120 Shimokuzawa, Sagamihara 229-1198, Japan

4 Graduate School of Pure and Applied Sciences,

University of Tsukuba, 1-1-1 Tennodai, Tsukuba 305-8571, Japan

5 Institute of Solid State Physics, University of Latvia, Kengaraga iela 8, LV1063 Riga, Latvia

6 Materials and Structures Laboratory $\&$ Frontier

Research Center, Tokyo Institute of Technology,

4259 Nagatsuta, Midori-ku, Yokohama 226-8503, Japan

* Author to whom correspondence should be addressed; e-mail: kkaji@tmu.ac.jp 


\begin{abstract}
Effects of fluorine doping on the diffusion of interstitial oxygen molecules $\left(\mathrm{O}_{2}\right)$ in amorphous $\mathrm{SiO}_{2}$ $\left(a-\mathrm{SiO}_{2}\right)$ were compared to those obtained from $a-\mathrm{SiO}_{2}$ containing $\mathrm{SiOH}$ groups. Incorporation of moderate concentration $\left(\sim 10^{19} \mathrm{~cm}^{-3}\right)$ of $\mathrm{SiF}$ groups gives rise to minor changes in diffusion parameters between 800 and $1100^{\circ} \mathrm{C}$ : only a slight decrease in solubility and an increase in the activation energy for diffusion can be detected. Incorporation of $\mathrm{SiOH}$ groups has similar weak effects on the solubility and activation energy for diffusion. These minor changes are most likely due to the enhancement of the flexibility of local Si-O network as a result of the dissociation of the network by $\mathrm{SiOH}$ and $\mathrm{SiF}$ groups. However, in contrast to the $\mathrm{SiF}$ doping, $\mathrm{SiOH}$ doping leads to a notable decrease in the diffusion coefficient. The heat of solution changes by $\sim 0.1-0.2 \mathrm{eV}$ at $\sim 1000^{\circ} \mathrm{C}$ and it is attributed to the glass transition of $a-\mathrm{SiO}_{2}$.
\end{abstract}




\section{INTRODUCTION}

Amorphous $\mathrm{SiO}_{2}\left(a-\mathrm{SiO}_{2}\right)$ is widely used as gate dielectric films for silicon microelectronic circuits, optical fibers for telecommunication, and optical components in excimer laser photolithography. Fluorine is one of the most important dopant for $a-\mathrm{SiO}_{2}$ used as such devices, because moderate fluorine doping increases the radiation hardness of $a-\mathrm{SiO}_{2}[1-4]$, suppresses the electrical breakdown of the gate dielectric films, and improves the optical transmittance near the absorption edge of $a-\mathrm{SiO}_{2}$ located at $h \nu \simeq 8 \mathrm{eV}$ [5]. These improvements are mainly due to the breaking up of Si-O network by Si-F bonds. It decreases the viscosity of $a-\mathrm{SiO}_{2}$ [3] and enhances the structural relaxation [6, 7], facilitating the removal of "strained" Si-O-Si bonds, which are considered to be a major source of point defects in $a-\mathrm{SiO}_{2}$ [8-13]. Furthermore, Si-F bonds themselves are stronger than $\mathrm{Si}-\mathrm{O}$ bonds that build the $a-\mathrm{SiO}_{2}$ network and are hardly decomposed. Thus, radiation hardness of fluorine-doped $a-\mathrm{SiO}_{2}$ is better than that of $a-\mathrm{SiO}_{2}$ containing other network modifiers, such as $\mathrm{SiOH}$ and $\mathrm{SiCl}$ groups. Similarly to $\mathrm{SiF}$ groups they enhance the structural relaxation, however, they can be converted to point defects under radiation or electrical stress.

Oxygen molecules dissolved in interstices of Si-O network (interstitial $\mathrm{O}_{2}$ ) are the main mobile oxygen species in $a-\mathrm{SiO}_{2}[14-16]$. They play a key role in thermal oxidation of silicon [17] and radiation induced defect processes in $a-\mathrm{SiO}_{2}$ [18]. Interstitial $\mathrm{O}_{2}$ in $a-\mathrm{SiO}_{2}$ are sensitively detected by their characteristic infrared photoluminescence at $\sim 1273 \mathrm{~nm}$, attributed to the transition from the lower excited singlet state $\left(a^{1} \Delta_{\mathrm{g}}\right)$ to the ground state $\left(X^{3} \Sigma_{g}^{-}\right)$[19]. It is possible to detect as few as $\sim 10^{14} \mathrm{~cm}^{-3}$ interstitial $\mathrm{O}_{2}$ when the upper excited singlet state $\left(b^{1} \Sigma_{\mathrm{g}}^{+}\right)$is populated using a continuous-wave laser light at a wavelength of $765 \mathrm{~nm}$ [20]. The sensitivity is sufficient to detect interstitial $\mathrm{O}_{2}$ incorporated during thermal annealing in air [21], offering an easy and straightforward way to quantitatively study the thermal diffusion of interstitial $\mathrm{O}_{2}$ in $a-\mathrm{SiO}_{2}$ [22]. Furthermore, this PL method is precise enough to evaluate the variations of the solubility and diffusion coefficient of interstitial $\mathrm{O}_{2}$ with the incorporation of $\sim 10^{20} \mathrm{~cm}^{-3} \mathrm{SiOH}$ groups $[18,23]$, which are the most common network modifiers in synthetic $a-\mathrm{SiO}_{2}$.

The purpose of the present study is to examine the influence of the incorporation of $\mathrm{SiF}$ groups on the diffusion of interstitial $\mathrm{O}_{2}$ in $a-\mathrm{SiO}_{2}$ and to compare it with that of $\mathrm{SiOH}$ groups. 


\section{EXPERIMENTAL PROCEDURE}

Fluorine-doped synthetic $\mathrm{SiO}_{2}$ glass containing $\sim 1.4 \times 10^{19} \mathrm{~cm}^{-3}$ of $\mathrm{SiF}$ groups and $\sim 1-2 \times 10^{18} \mathrm{~cm}^{-3}$ of $\mathrm{SiOH}$ groups was cut into specimens in the form of $7 \times 10 \times 1 \mathrm{~mm}^{3}$, and the two largest faces were polished to an optical finish. They were thermally annealed in air at $800,900,1000$, or $1100^{\circ} \mathrm{C}$ to incorporate interstitial $\mathrm{O}_{2}$. The PL band of interstitial $\mathrm{O}_{2}$ in the $\mathrm{O}_{2}$-loaded samples was excited at $765 \mathrm{~nm}$ using an AlGaAs laser diode $(\sim 1.5 \mathrm{~W}$ at the sample position) and was measured using the detector part of a Fourier-transform Raman spectrometer (Model 960 Nicolet). The laser light was directed normal to the polished surface and the backscattered PL signal was recorded. The peak amplitude of the $\mathrm{PL}$ band is proportional to the thickness average of the concentration of interstitial $\mathrm{O}_{2}, C_{\mathrm{a}}$, and the proportionality factor was determined using a reference sample with a known $\mathrm{O}_{2}$ concentration.

\section{RESULTS}

Figure 1 shows the variation of $C_{\mathrm{a}}$ with annealing time $t$ at $800,900,1000$, or $1100^{\circ} \mathrm{C}$. $C_{\mathrm{a}}$ was proportional to $t^{1 / 2}$ at small $t$, and saturated at a constant value at large $t$. This observation indicates that the dissolution of $\mathrm{O}_{2}$ from air is much faster than the following $\mathrm{O}_{2}$ diffusion in $a-\mathrm{SiO}_{2}[22]$ and is consistent with previous results $[22,23]$. Thus, the observed variation of $C_{\mathrm{a}}$ with $t$ was simulated well by an equation describing the simplest one-dimensional diffusion in a parallel sheet of a thickness $L$ [24],

$$
\frac{C_{\mathrm{a}}(t)}{C_{0}}=1-\frac{8}{\pi^{2}} \sum_{n=1}^{\infty} \frac{\exp \left[-D(2 n-1)^{2} \pi^{2} t / L^{2}\right]}{(2 n-1)^{2}}
$$

using the diffusion coefficient $D$ and the saturation concentration $C_{0}$ as adjustable parameters. The best-fit theoretical curves are shown as solid lines in Fig. 1. The solubility $S$ was calculated from the relation $S=C_{0} / p_{\mathrm{O}_{2}}$, where $p_{\mathrm{O}_{2}}$ is the partial pressure of $\mathrm{O}_{2}$ in air at atmospheric pressure $\left(p_{\mathrm{O}_{2}}=0.209 \mathrm{~atm}\right)$, provided that $C_{0}$ is proportional to $p_{\mathrm{O}_{2}}$ at this pressure range [15, 23].

The $D$ and $S$ values derived from the data shown in Fig. 1 are plotted in Fig. 2 and are referred to as "F-doped". Both $\log D$ and $\log S$ were almost proportional to the reciprocal of the absolute temperature $T$, with slight deviations starting to appear above $\sim 1000^{\circ} \mathrm{C}$ in 
the $S$ plot. Apart from the point at $1100^{\circ} \mathrm{C}$ in the $S$ plot, these data were fitted to the simple Arrhenius-type relations

$$
\begin{gathered}
D=D_{0} \exp \left(-\Delta E_{\mathrm{a}} / k T\right), \\
S=S_{0} \exp (-\Delta H / k T),
\end{gathered}
$$

to evaluate the activation energy for diffusion $\Delta E_{\mathrm{a}}$, the heat of solution $\Delta H$, and the preexponential factors $D_{0}$ and $S_{0} . k$ in Eqs. (2) and (3) denotes the Boltzmann constant. The calculated parameters are listed in Table I. The experimental uncertainties of $\Delta E_{\mathrm{a}}$ and $D_{0}$ were larger for the F-doped sample than for the LowOH and HighOH samples reported previously [18, 23], because the temperature range used for the fitting of the F-doped sample data was narrower.

\section{DISCUSSION}

Figure 2 also shows $D$ and $S$ values of interstitial $\mathrm{O}_{2}$ reported to date. The obtained $\Delta E_{\mathrm{a}}, \Delta H, D_{0}$, and $S_{0}$ values are listed in Table I, along with the measurement method, sample type, and abbreviated name. Agreements among data are good for $D, \Delta E_{\mathrm{a}}$, and $\Delta H$. However, our $S$ data are $\sim 2.5$ times smaller than those reported in Ref. [15].

The Low $\mathrm{OH}$ sample is fluorine-free and contains $\mathrm{SiOH}$ groups in concentration comparable with that in the F-doped sample. In these two samples the behavior of diffusion of interstitial $\mathrm{O}_{2}$ is very similar, except for a subtly higher $\Delta E_{\mathrm{a}}$ and smaller $S$ in the F-doped sample. This observation indicates that incorporation of $\sim 10^{19} \mathrm{~cm}^{-3} \mathrm{SiF}$ groups does not significantly modify the diffusion of interstitial $\mathrm{O}_{2}$.

The diffusion of molecular species in solids has been studied well for amorphous organic polymers, and these results may provide insight into the diffusion of molecular species in $a-\mathrm{SiO}_{2}$. In amorphous organic polymers, $\Delta H$ above the glass transition temperature $\left(T_{\mathrm{g}}\right)$ is generally larger (i.e., smaller by absolute value since $\Delta H$ is negative) than that below $T_{\mathrm{g}}[25]$. It is explained by postulating that the main dissolution mechanism below $T_{\mathrm{g}}$ is exothermic trapping of molecules in relatively rigid interstitial voids. Above $T_{\mathrm{g}}$, these voids are considered to be destroyed by the enhancement of the thermal motion of the network, indicating that heat to form voids is additionally needed to incorporate molecules. In $a$ $\mathrm{SiO}_{2}$, a change in $\Delta H$ is observed at $\sim 1000^{\circ} \mathrm{C}$ in all samples we measured [Fig. 2(b)]. All 
these samples became deformed by viscous flow at $1200^{\circ} \mathrm{C}$, indicating that $T_{\mathrm{g}}$ is lower than $1200^{\circ} \mathrm{C}$. The measurements of the specific volume [26], the heat capacity [26, 27], and the light scattering intensity [28] show that $T_{\mathrm{g}}$ of $a-\mathrm{SiO}_{2}$ is $\sim 1000-1200^{\circ} \mathrm{C}$, and that $T_{\mathrm{g}}$ decreases with an increase in the concentration of the network modifiers [28]. Although the reported $T_{\mathrm{g}}$ values are slightly higher than the observed change in $\Delta H$ at $\sim 1000^{\circ} \mathrm{C}$, we suggest that the $\Delta H$ change is due to the glass transition of $a-\mathrm{SiO}_{2}$. Similar temperature difference can be observed for organic polymers with multiple relaxation processes [29]. Thus, the $\Delta H$ change may be related to structural rearrangements of local network domains (subrelaxation) below $T_{\mathrm{g}}$, which is promoted by the presence of network modifiers, such as $\mathrm{SiOH}, \mathrm{SiF}$, and $\mathrm{SiCl}$ groups $[30,31]$.

The network modifiers dissociate the Si-O network and increase its flexibility, thus enhancing the local thermal motion around them [28]. This enhanced local thermal motion may collapse interstitial voids, with a mechanism similar to the destruction of the voids above $T_{\mathrm{g}}$. The total concentration of the network modifiers in our samples increases in the order of LowOH, F-doped, and HighOH. This mechanism may explain the observed decrease in $S$ in this order.

In amorphous organic polymers $\Delta E_{\mathrm{a}}$ above $T_{\mathrm{g}}$ is generally larger than that below $T_{\mathrm{g}}$. It is attributed to a larger jump distance of a molecule above $T_{\mathrm{g}}$. A theory that describes the diffusion based on this mechanism predicts that the increase in $\Delta E_{\mathrm{a}}$ is equal to $\Delta H$ above $T_{\mathrm{g}}$ minus $\Delta H$ below $T_{\mathrm{g}}[32]$. However, the changes in $\Delta E_{\mathrm{a}}$ actually observed for the diffusion of $\mathrm{O}_{2}$ in poly(vinyl acetate) [33] and poly(ethylene terephthalate) [32] are $\sim 1 / 2$ of those expected by the theory. If similar mechanism were effective in $a-\mathrm{SiO}_{2}$, the change in $\Delta E_{\mathrm{a}}$ at $T_{\mathrm{g}}$ would be $\sim 0.1 \mathrm{eV}$. Considering the number of the data points and the experimental uncertainty shown in Fig. 2(a), the changes in $\Delta E_{\mathrm{a}}$ at $T_{\mathrm{g}}$ are no larger than $\sim 0.1 \mathrm{eV}$ in $a-\mathrm{SiO}_{2}$ samples we measured. However, changes in $\Delta E_{\mathrm{a}}$ less than $\sim 0.1 \mathrm{eV}$ cannot be excluded. Nonetheless, the observed increase in $\Delta E_{\mathrm{a}}$ with an increase in the concentrations of the network modifiers may be related the increase in the network flexibility caused by the incorporation of the network modifiers. Similar increase in $\Delta E_{\mathrm{a}}$ with an increase in the concentration of $\mathrm{SiOH}$ groups is observed for the diffusion of $\mathrm{He}$ in $a-\mathrm{SiO}_{2}$ [34].

Although $\mathrm{SiF}$ and $\mathrm{SiOH}$ groups play qualitatively similar roles in the dissolution and diffusion of interstitial $\mathrm{O}_{2}$ as described above, their quantitative effect on $D$ (i.e. dependence of $D$ on the concentration of network modifiers) seems different. $D$ of the F-doped sample 
is almost equal to that of the Low $\mathrm{OH}$ samples between 800 and $1100^{\circ} \mathrm{C}$, whereas $\mathrm{D}$ of the HighOH sample is markedly smaller. Although the difference may be partly due to the smaller $(\sim 1 / 7)$ concentration of SiF groups in the F-doped sample than that of $\mathrm{SiOH}$ groups in the HighOH sample, these data imply that $\mathrm{SiOH}$ groups disturb the diffusion of interstitial $\mathrm{O}_{2}$ more strongly than $\mathrm{SiF}$ groups. This difference can be attributed to the difference in chemical properties between $\mathrm{SiOH}$ and $\mathrm{SiF}$ groups, most likely the higher chemical activity of $\mathrm{SiOH}$ groups, such as their ability to form hydrogen bonds and participation in hydrationdehydration reactions. However, further studies are obviously needed to test this hypothesis.

\section{CONCLUSIONS}

Diffusion of interstitial oxygen molecules $\left(\mathrm{O}_{2}\right)$ in fluorine-doped synthetic amorphous $\mathrm{SiO}_{2}\left(a-\mathrm{SiO}_{2}\right)$ was examined. The results are compared with the data taken from $a-\mathrm{SiO}_{2}$ containing $\mathrm{SiOH}$ groups and are analyzed in terms of the concentration of network modifiers ( $\mathrm{SiOH}$ and $\mathrm{SiF}$ groups). The observations indicate that solubility decreases and the activation energy for diffusion increases with an increase in the concentration of network modifiers. A comparison of the results with those reported for amorphous organic polymers suggests that such changes in the solubility and the activation energy for diffusion are attributed to the promotion of the local thermal motion of the Si-O network, resulting from

the incorporation of network modifiers that dissociates the Si-O network. The change of the heat of solution at $\sim 1000^{\circ} \mathrm{C}$ is probably due to the glass transition of $a-\mathrm{SiO}_{2}$, where the temperature dependence of the local thermal motion is expected to change discontinuously. However, change in the activation energy for diffusion at the glass transition temperature was not above experimental uncertainty. The diffusion coefficient is decreased by SiOH doping whereas it is varied only slightly by $\mathrm{SiF}$ doping, possibly because $\mathrm{SiOH}$ groups are more chemically active than $\mathrm{SiF}$ groups. 
[1] K. Awazu, H. Kawazoe, and K. Muta, J. Appl. Phys. 69, 4183 (1991).

[2] K. Arai, H. Imai, J. Isoya, H. Hosono, Y. Abe, and H. Imagawa, Phys. Rev. B 45, 10818 (1992).

[3] M. Kyoto, Y. Ohoga, S. Ishikawa, and Y. Ishiguro, J. Mater. Sci. 28, 2738 (1993).

[4] K. Kajihara, M. Hirano, L. Skuja, and H. Hosono, Mater. Sci. Eng. B 161, 96 (2009).

[5] H. Hosono, M. Mizuguchi, H. Kawazoe, and T. Ogawa, Appl. Phys. Lett. 74, 2755 (1999).

[6] Y. Ikuta, S. Kikugawa, M. Hirano, and H. Hosono, J. Vac. Sci. Technol. B 18, 2891 (2000).

[7] K. Saito and A. J. Ikushima, J. Appl. Phys. 91, 4886 (2002).

[8] R. A. B. Devine and J. Arndt, Phys. Rev. B 39, 5132 (1989).

[9] R. A. B. Devine and J. Arndt, Phys. Rev. B 42, 2617 (1990).

[10] H. Imai, K. Arai, J. Isoya, H. Hosono, Y. Abe, and H. Imagawa, Phys. Rev. B 48, 3116 (1993).

[11] H. Hosono, Y. Ikuta, T. Kinoshita, K. Kajihara, and M. Hirano, Phys. Rev. Lett. 87, 175501 (2001).

[12] K. Awazu and H. Kawazoe, J. Appl. Phys. 94, 6243 (2003).

[13] K. Kajihara, M. Hirano, L. Skuja, and H. Hosono, Phys. Rev. B 78, 094201 (2008).

[14] R. H. Doremus, Diffusion of Reactive Molecules in Solids and Melts (John Wiley \& Sons, New York, 2002).

[15] F. J. Norton, Nature 191, 701 (1961).

[16] M. A. Lamkin, F. L. Riley, and R. J. Fordham, J. Eur. Ceram. Soc. 10, 347 (1992).

[17] B. E. Deal and A. S. Grove, J. Appl. Phys. 36, 3770 (1965).

[18] K. Kajihara, T. Miura, H. Kamioka, A. Aiba, M. Uramoto, Y. Morimoto, M. Hirano, L. Skuja, and H. Hosono, J. Non-Cryst. Solids 354, 224 (2008).

[19] L. Skuja and B. Güttler, Phys. Rev. Lett. 77, 2093 (1996).

[20] L. Skuja, B. Güttler, D. Schiel, and A. R. Silin, Phys. Rev. B 58, 14296 (1998).

[21] K. Kajihara, T. Miura, H. Kamioka, M. Hirano, L. Skuja, and H. Hosono, J. Non-Cryst. Solids 349, 205 (2004).

[22] K. Kajihara, T. Miura, H. Kamioka, M. Hirano, L. Skuja, and H. Hosono, J. Ceram. Soc. Jpn. 112, 559 (2004).

[23] K. Kajihara, H. Kamioka, M. Hirano, T. Miura, L. Skuja, and H. Hosono, J. Appl. Phys. 98, 
013529 (2005).

[24] J. Crank, The Mathematics of Diffusion, 2 ed. (Oxford University Press, Oxford, 1975).

[25] A. S. Michaels, W. R. Vieth, and J. A. Barrie, J. Appl. Phys. 34, 1 (1963).

[26] R. Brückner, J. Non-Cryst. Solids 5, 123 (1970).

[27] P. Richet and Y. Bottinga, Geochim. Cosmochim. Acta 48, 453 (1984).

[28] K. Saito and A. J. Ikushima, Appl. Phys. Lett. 70, 3504 (1997).

[29] Y. P. Yampolskii, Y. Kamiya, and A. Y. Alentiev, J. Appl. Polym. Sci. 76, 1691 (2000).

[30] A. J. Ikushima, T. Fujiwara, and K. Saito, J. Appl. Phys. 88, 1201 (2000).

[31] K. Saito and A. J. Ikushima, Appl. Phys. Lett. 73, 1209 (1998).

[32] A. S. Michaels, W. R. Vieth, and J. A. Barrie, J. Appl. Phys. 34, 13 (1963).

[33] P. Meares, J. Am. Chem. Soc. 76, 3415 (1954).

[34] J. E. Shelby, J. Am. Ceram. Soc. 55, 61 (1972).

[35] G. Hetherington and K. H. Jack, Phys. Chem. Glasses 5, 147 (1964).

[36] C. C. Tournour and J. E. Shelby, Phys. Chem. Glasses 46, 559 (2005).

[37] R. M. Barrer, J. Chem. Soc. 136, 378 (1934). 
FIG. 1: Variation of the thickness average of interstitial $\mathrm{O}_{2}$ concentration $C_{\mathrm{a}}$ with annealing time $t$ for $1 \mathrm{~mm}$-thick samples annealed in air at $800,900,1000$, or $1100^{\circ} \mathrm{C}$. The error bar indicates the experimental uncertainty. The inset shows the PL band of interstitial $\mathrm{O}_{2}$ for samples annealed at $900^{\circ} \mathrm{C}$.

FIG. 2: Arrhenius plots of the diffusion coefficient (a) and solubility (b) of interstitial $\mathrm{O}_{2}$. Data taken from Refs. $[15,23,35,36]$ are also shown. The error bars indicate the experimental uncertainty for data obtained by our group (F-doped, LowOH, and HighOH). The dotted lines at $T \geqq 1000^{\circ} \mathrm{C}$ in panel (b) correspond to linear fits drawn to guide the eye. 


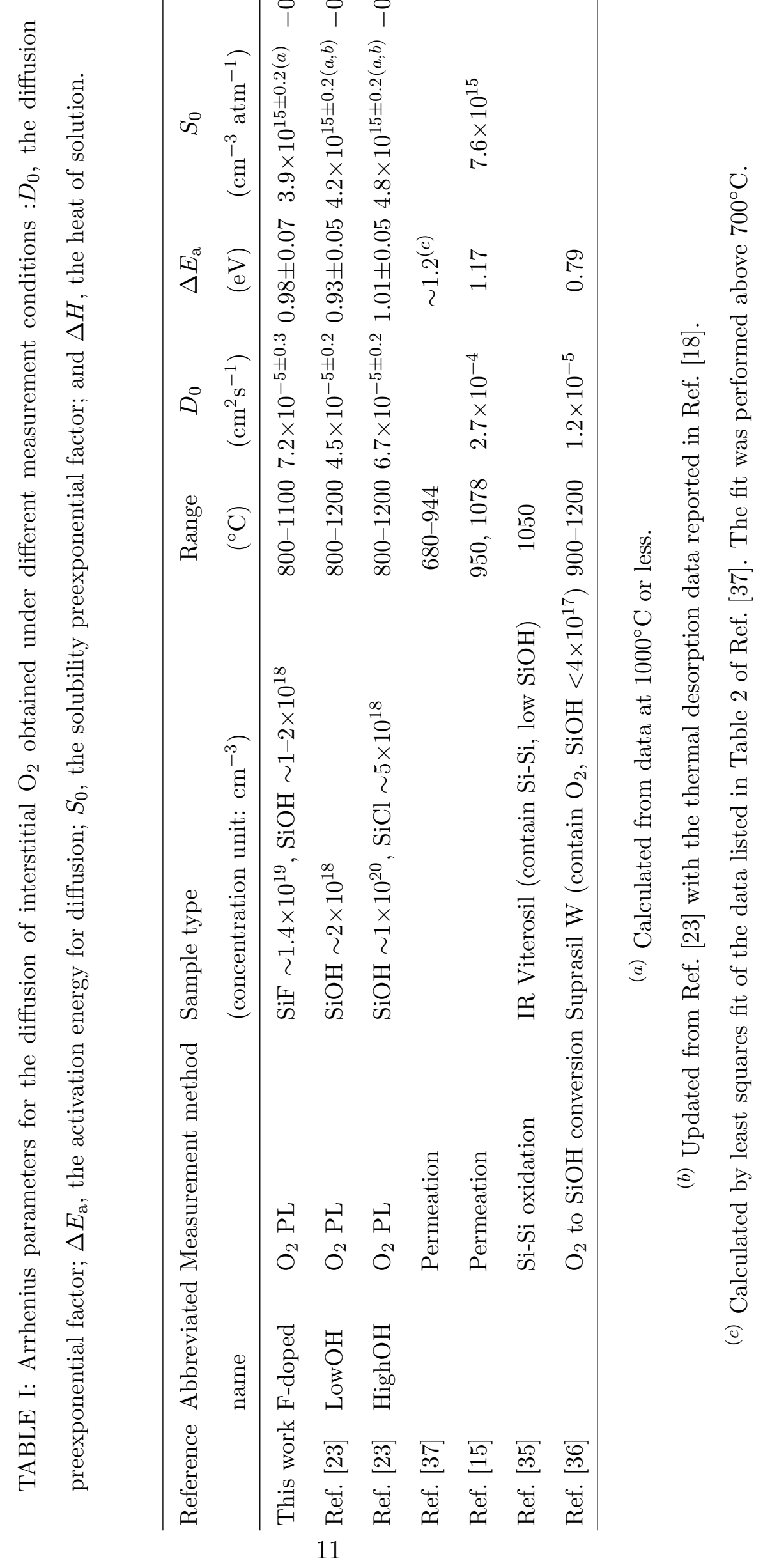




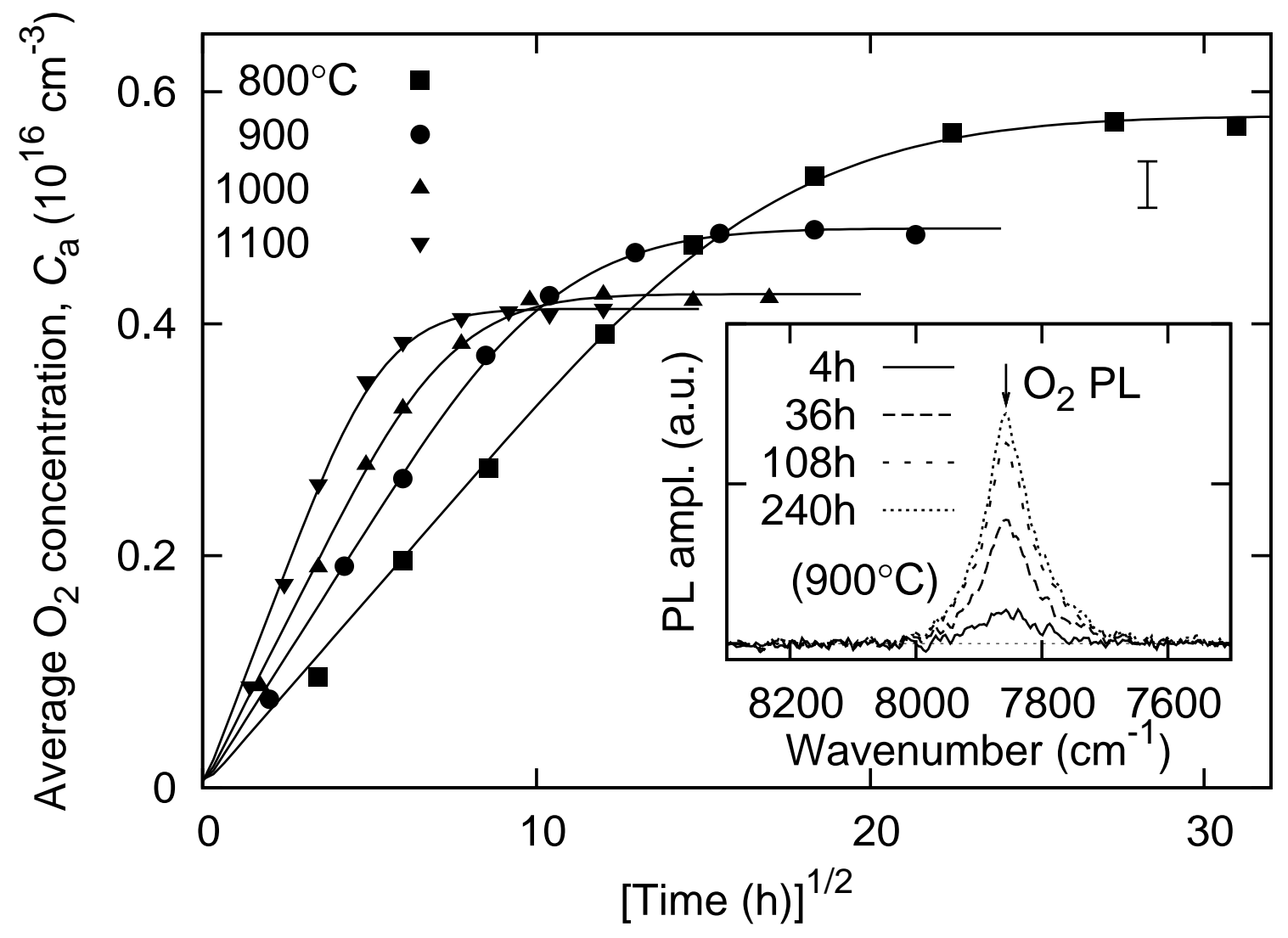

Fig.1 K. Kajihara et al. 

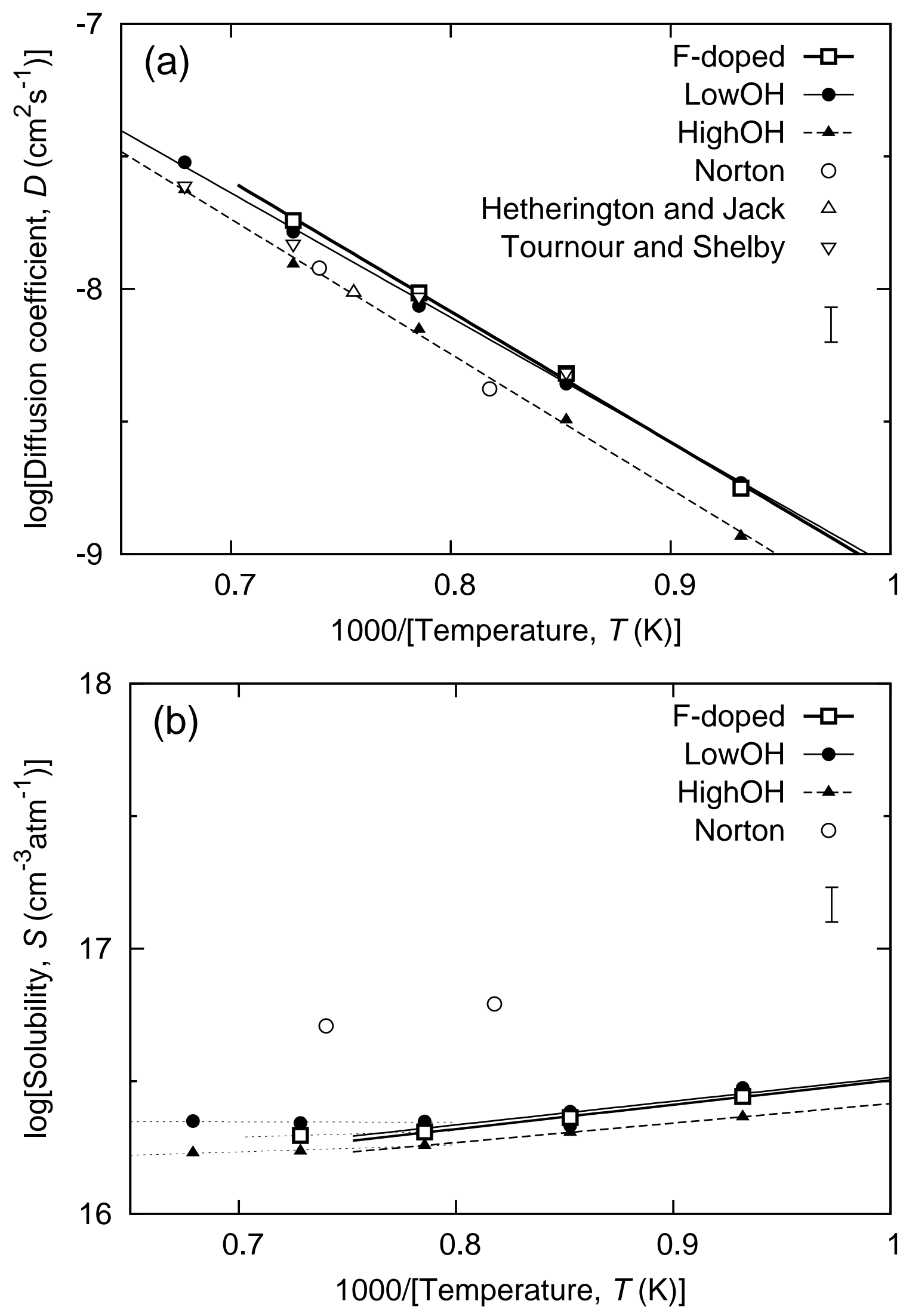

Fig.2 K. Kajihara et al. 